\title{
Annealing Effects on the Properties of Copper Selenide Thin Films for Thermoelectric Applications
}

\author{
Desapogu Rajesh*, R Rajesh Chandrakanth and C S Sunandana* \\ School of Physics, University of Hyderabad, Hyderabad - 500046, A.P; INDIA
}

\begin{abstract}
Recent results concerning structural and optical features of the thermal evaporated CuSe thin nanoparticulatefilms are presented. The preparative parameters were optimized to obtain good quality of thin films. Structure and properties of materials were characterized with XRD and optical spectroscopy and Field emission scanning electron microscopy (FESEM), Atomic force microscopy. CuSe pelletspossess an electrical resistivity (4 - point probe) of $3.156 \mu \Omega m$ at $300 \mathrm{~K}$. The figure of merit is calculated as 1.34 .
\end{abstract}

\section{Introduction}

Highly efficient thermoelectric materials have attracted remendo us attention because of various technological applications such as power generation from waste heat and environmentally friendly refrigeration [1-3]. Coppers elenide has been studied with great interest during the past decades because of its potential applications in in thermo electric materials and various methods have been applied to prepare these important nano crystals, such as solve thermal method [4,5], $\gamma$-irradiation route [6], Microwave-assisted heating method [7]. However, only a few studies on the phase control of nano crystal line coppers elenide have been reported. For example, the effect of solvent and surfactant has been investigated in the formation of different phases of nano crystal line coppers elenide by Xie and co-workers using as on chemical approach [8]. Therefore, further study the phase control of nano crystal line coppers elenide with simple process would be interesting.

Herein, we report a novel and convenient route to CuSe thin films by thermal evaporation. Further, we present results on annealing effects of CuSe thin films.

\section{Sample preparation}

The elemental form of Copper and Selenium were taken in powder form in the ratio 1:1 by weight. Then it was grinded in an agate motor for $1 \mathrm{hr}$ at room temperature to form copper selenide. The whole sample was divided into two parts. One part of the sample was used to make pellets using hydraulic press. Another part of the sample was used to make thin films on a glass substrate by thermal evaporation using molybdenum boat in vacuum. Then the as deposited thin films were cut into small pieces. These small pieces are annealed at 200 ${ }^{0} \mathrm{C}, 300{ }^{0} \mathrm{C}, 5000 \mathrm{C}$ and $7000 \mathrm{C}$ for 30 minutes.

XRD were recorded for the CuSe powder, pellets, as deposited film and for the films annealed at different temperatures. The pellet was used to determine the resistivity of the sample by four probe method. Absorption spectra were recorded for all the thin film samples by UV-VIS spectroscopy. The surface property measurements were done using Field Emission Scanning Electron Microscopy (FESEM) and Atomic Force Microscopy (AFM).

\subsection{Structural studies}

\section{Results and Discussions}

XRD patterns of the CuSe films annealed at $200^{\circ} \mathrm{C}$ and $300^{\circ} \mathrm{C}$ along with that of an as - deposited film are shown in fig 1 . As deposited CuSe thin film shows the hexagonal structure with well-defined peak at $36.65^{\circ}$ corresponding to reflection from (115)plane (JCPDS 89-2734), as observed in fig 1a. The Copper Selenide thin films annealed at $200{ }^{\circ} \mathrm{C}$ for 30 minutes shows the hexagonal structure with well-defined peaks at $23.73{ }^{\circ}, 30.03$ , $31.39^{\circ}, 34.59^{\circ}, 44.00^{\circ}$, and $55.97^{\circ}$ corresponding to the (004), (310), (311), (304), (330) and (252) planes respectively (JCPDS 89-2734) are observed in fig $1 \mathrm{~b}$. The Bragg reflections increased in intensity after annealing at the $200^{\circ} \mathrm{C}$. A high intensity peak is observed at $31.77^{\circ}$ for thin film annealed at $300{ }^{\circ} \mathrm{C}$ from the plane (311). The full width at half maximum (FWHM) of the peak decreased drasticallyupon increases of annealing temperaturefrom $200{ }^{\circ} \mathrm{C}$ to $300{ }^{\circ} \mathrm{C}$ indicating that there is an increase in the crystalline nature of the Copper selenide films upon increasing annealing temperature. Whereas, no peaks were observed for thin films annealed at 500 and $700{ }^{\circ} \mathrm{C}$. The crystallite sizes were calculated for the samples using Scherer's formula, $D=\frac{0.93 \lambda}{\beta \cos (i \theta)}$ and the lattice parameters (a and c) were calculated using the relation,

$$
\frac{1}{d^{2}}=\frac{3}{4}\left[\frac{h^{2}+k^{2}-h k}{a^{2}}+\frac{3 l^{2}}{4 c^{2}}-\frac{(k+h) l}{a c}\right]
$$


Whereh, k, 1 are the miller indices for corresponding planes. Crystallite sizes and lattice parameters at various temperatures are presented in the table 1 . We observed that crystallite size is increasing with annealing temperature.

$\mathrm{Cu}$, Se powders were taken in $\mathrm{Cu}$ : Se (1:1) ratio and thoroughly grinded for 30 min by mechano-chemical method. We tested it with the formation of $\mathrm{CuSe}$ (figure 3.2.) by comparing the peaks with the standard data (JCPDS 89-2734) and found to be in hexagonal phase. Also, we made a pellet for resistivity measurement, which is also showing the CuSe formation shown in the fig 3 .

\subsection{Optical properties}

The absorption spectra of copper selenide thin films of as-deposited and annealed at various temperatures $\left(200{ }^{\circ} \mathrm{C}, 300{ }^{\circ} \mathrm{C}, 500{ }^{\circ} \mathrm{C}\right.$ and $\left.700{ }^{\circ} \mathrm{C}\right)$ were shown in the fig 4 . We observed that there is a decrease in the intensity of absorption spectra of annealed films. The absorption spectra are temperature independent, confirming that $\mathrm{CuSe}$ thin films show direct transition. A blue shift of absorption spectrum is observed with increase in annealing temperature, indicating that band gap increase with annealing temperature. The thickness and band gaps of as-deposited and annealed CuSe thin films are presented in the table 2. Variation of thickness and band gap of as-deposited and annealed CuSe thin films with temperature is shown in fig 5. The thickness of the $\mathrm{CuSe}$ thin films is decreasing with temperature whereas band gap increases with annealing temperature, confirming the blue shift of absorption spectra shown in fig4.

Because of the crystallinity and higher transparency, the Copper selenide films are suitable for optical analysis from which the absorption coefficient and energy band gap may be determined. In the fundamental absorption region the optical absorption coefficient, $\alpha$ was evaluated using the relation, $\alpha=\left(\ln \mathrm{A}^{-1}\right) / t$, where $t$ is the film thickness and $A$ is the absorbance. The best linear relationship is obtained by plotting $\alpha^{1 / 2}$ against $h v$, based on the below equation,

$$
\alpha h v=A(h v-E g)^{n / 2}
$$

Where, $\alpha$ is absorption coefficient, $A$ is constant (independent of $v$ ) and $n$ is the exponent that depends on the quantum selection rules for the particular material. The photon energy (hv) for $\mathrm{x}$-axis can be calculated using below equqtion,

$$
\mathbf{E}=\mathbf{h v}=\mathbf{h c} / \boldsymbol{\lambda}
$$

Where, $h$ is Planck's constant $\left(6.626 \times 10^{-34} \mathrm{Js}\right), c$ is speed of light $\left(3 \times 10^{8} \mathrm{~m} / \mathrm{s}\right)$ and $\lambda$ is the wavelength of the light used.With increase in the annealing temperature, the thickness of the film was decreasing. However, the corresponding band gap was increasing with temperature. The same was presented in the fig 5 .

\subsection{Surface analysis}

The morphology of the films was substantially affected by the annealing temperature. The as-deposited film, without annealing, has a rough surface and irregular grain size as observed in fig6a. When the films were annealed at $200{ }^{\circ} \mathrm{C}$ for 30 minutes, the morphology was not changed much but showed better uniformity in grain size (fig 6 b). The annealed film at $300{ }^{\circ} \mathrm{C}$ shows a continuous and homogeneous granular surface as observed in fig6c. The morphology showed dense spherical structures and no visible defects indicate that the films are completely covered with particles. And figures $6 \mathrm{~d}, 6$ e and $6 \mathrm{f}$ are clearly shows the particle size was increased with annealing temperature.

The grain sizes and RMS roughness of as such and annealed films were shown in the table 3 . The random distribution of grains, in projection and size, only suggests a random nucleation mechanism and random orientation of grains show that the grain growth is isotropic. The dependence of grain size, RMS roughness on temperature is demonstrated in the figure 7 . The grain size increased more rapidly with the annealing temperature and the grain growth rate increases more rapidly at higher annealing temperature. This kinetics of grain growth trend was influenced by the grain size which is in agreement with Huda and Ralph [1].

\subsection{FESEM}

Figure 8a shows FESEM image of as deposited CuSe film deposited on borosilicate glass substrate and annealed at $200{ }^{\circ} \mathrm{C}, 500{ }^{\circ} \mathrm{C}$ for 30 minutes. It can be clearly seen that the copper selenide surface is smooth and high dense with uniform cracks. The crystal grains of the copper selenide film are uniformly distributed on the surface and grain size is about $30-50 \mathrm{~nm}$, indicating that the copper selenide film has a well-defined surface morphology. The figures, $8 \mathrm{~b}$ and $8 \mathrm{c}$ show the morphology and the fully spherical nanoparticles with well separation in Copper selenide films annealed at $200{ }^{\circ} \mathrm{C}$ and $500{ }^{\circ} \mathrm{C}$ for 30 minutes. In XRD, optical results clearly agree with this morphological changes. In case of Figure $8 \mathrm{~d}$ the partially cover with flakes type morphology with high porosity happen in the films due to the annealing effect at $500{ }^{\circ} \mathrm{C}$. But in case of pellet form, a beautiful disk type micro structure has seen in figure 8e. The Electron dispersive scattering (EDS) spectrum in figure $8 \mathrm{f}$ clearly shows the elemental analysis of $\mathrm{CuSe}$ formation with high purity with 1:1 stoichiometry ratio. 


\subsection{Resistivity and Figure of Merit (ZT)}

The temperature dependence of resistivity is shown in figure 3.9. The resistivity of copper selenide increases with temperature and at room temperature $(300 \mathrm{~K})$, resistivity is measured as $3.156 \mu \Omega \mathrm{m}$. Due to various reasons, we could not measure Seebeck coefficient and thermal conductivity of the sample. However, to get a feel we have taken the above said values from literature[9] to calculate figure of merit of the sample. At room temperature $(300 \mathrm{~K})$, the Seebeck coefficient and thermal conductivity values are taken as $75 \mu \mathrm{V} / / \mathrm{K}$ and $0.4 \mathrm{Wm}^{-1} \mathrm{~K}^{-1}$ respectively. Hence, figure of merit is calculated as 1.34 at $300 \mathrm{~K}$. Also, the emphasis has to be made in preparing nano particles, which greatly reduces the thermal conductivity, thereby increasing the figure of merit. The $\mathrm{Cu}_{\mathrm{x}} \mathrm{Se}_{1-\mathrm{x}}$ stoichiometry ratios for improved figure of merit is under investigation.

\section{Conclusions}

XRD and AFM studies show that the crystallite size of Copper Selenide increases with temperature and spectroscopic studies tell that the band gap increases with the annealing temperature. The high band gap materials are employed in high temperature applications. The figure of merit for the sample is calculated as 1.34 at $300 \mathrm{~K}$, which is high compared to other thermoelectric materials.

\section{Acknowledgement:}

DR \& RRC thanks to School of Physics - University of Hyderabad for providing goodexperimental facilities. DR specially thanks to UGC-BSR for financial support.

\section{References:}

[1] M. S. Dresselhaus, G. Chen, M. Y. Tang, R. G.Yang, H. Lee, D. Z. Wang, Z. Ren, J. P. Fleurial, P. Gogna,Adv.Mater.2007,19, 1043 ;

[2] F. J. DiSalvo, Science1999, 285, 703

[3] J. R. Sootsman, D. Y. Chung, M. G. Kanatzidis,Angew. Chem.2009, 121, 8768; Angew. Chem. Int. Ed.2009, 48, 8616

[4] W.X. Zhang, X.M. Zhang, L. Zhang, J.X. Wu, Z.H. Hui,Y.W. Cheng,J.W. Liu, Y. Xie, and Y.T. Qian, Inorg.Chem. 39 (2000)1838.

[5] W. Z. Wang, P. Yan,F.Y. Liu, Y. Xie, Y. Geng and Y.T. Qian, J. Mater.Chem. 8 (1998)2321.

[6] Z.P. Qiao, Y. Xie, J.G. Xu, X.M. Liu, Y.J. Zhu and Y.T. Qian, Can.J.Chem. 78 (2000)1143.

[7] J.J. Zhu, O.Palchik, S.G. Chen and A. Gedanken, J. Phys.Chem. B 104 (2000)7344.

[8] Hong-Liang Li et al., J. Mat. Che. 12 (2002) 3723-3727.

[9]. B. Yu, et al.,'Thermoelectric properties of copper selenide with ordered selenium layer and disordered copper layer", Nano Energy, 2012 .

\section{Figure Captions}

Fig. 1. X- raydiffractogram of as-deposited and annealed at $200{ }^{\circ} \mathrm{C} \& 300{ }^{\circ} \mathrm{C}$ of copper selenide thin films.

Fig. 2. X- raydiffractogram of Copper selenide powder

Fig. 3. X-raydiffractogram of Copper selenide pellet.

Fig. 4. Absorption spectra of as - deposited and annealed at $200{ }^{\circ} \mathrm{C}, 300{ }^{\circ} \mathrm{C}, 500{ }^{\circ} \mathrm{C} \& 700{ }^{\circ} \mathrm{C}$ copper selenide thin films.

Fig. 5. Variation of thickness and band gap of as-deposited and annealed CuSe thin films with temperature.

Fig. 6. AFM micrographs of copper selenide (a) as - deposited film, and thin films annealed at (b) $200{ }^{\circ} \mathrm{C}$ and (c) $300{ }^{\circ} \mathrm{C}$ (d) $500{ }^{\circ} \mathrm{C}$ (e) $700{ }^{\circ} \mathrm{C}$ (f) 3-dimensional view of fig 6 e.

Fig. 7. Variation of crystallite size and RMS roughness of surface of CuSe films with temperature.

Fig. 8. FESEM micrographs of (a) as-deposited film, annealed at (b) $200{ }^{\circ} \mathrm{C}$ and (c) $500{ }^{\circ} \mathrm{C}$ (d) $700{ }^{\circ} \mathrm{C}$ copper selenide thin films (e) pellet and (f) EDS spectrum of the compound.

Fig. 9.Temperature dependence of resistivity.

Table

Table 1. Microstructural parameters associated with the respective samples.

Table 2. Thickness and band gaps of as-deposited and annealed Copper selenide thin films.

Table 3. Crystallite size and RMS roughness of as-deposited and annealed films. 


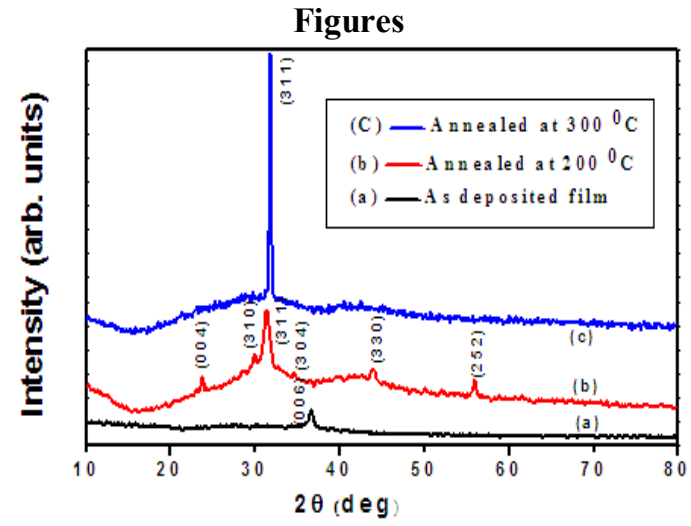

Figure 1

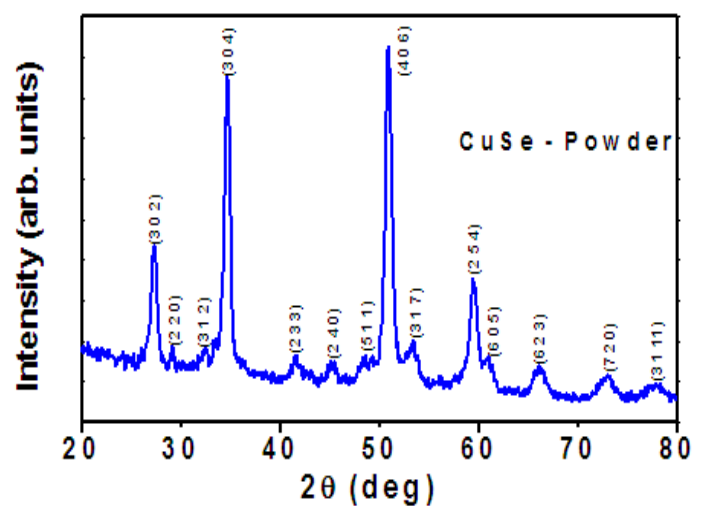

Figure 2

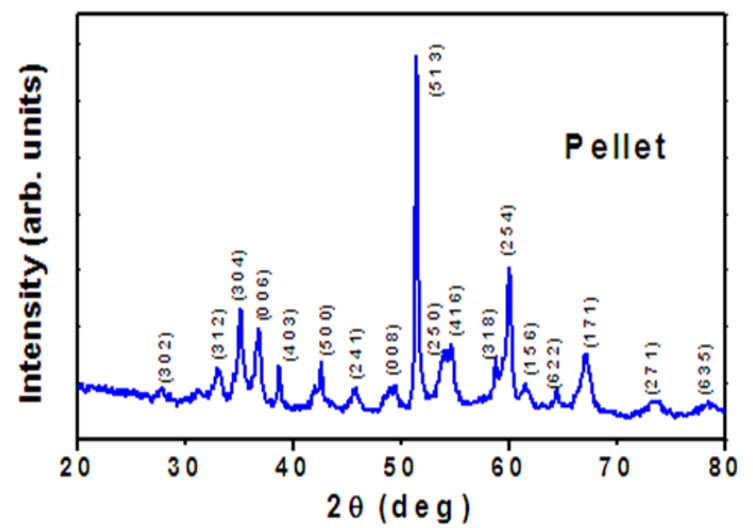

Figure 3

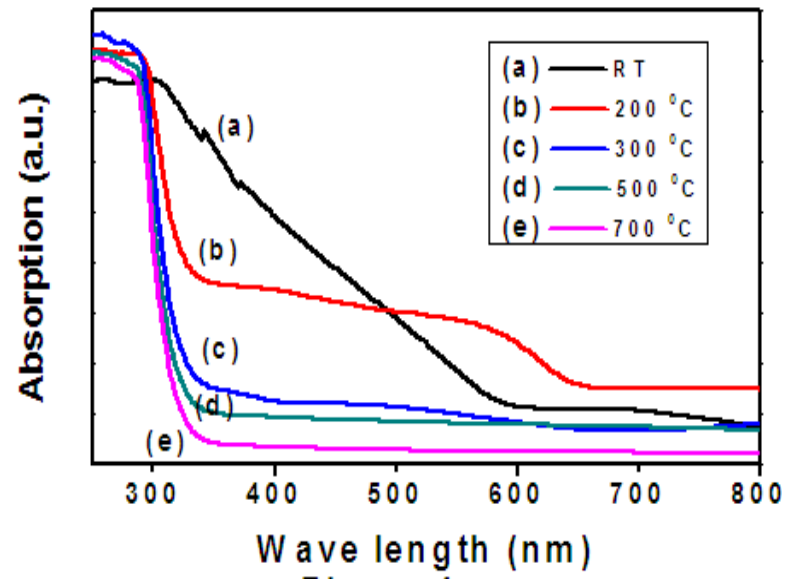

Figure 4 


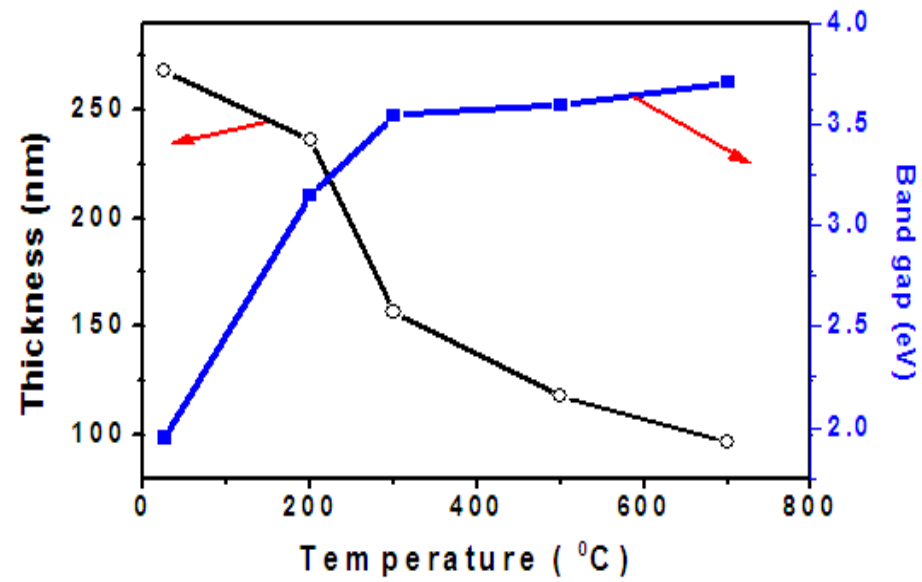

Figure 5
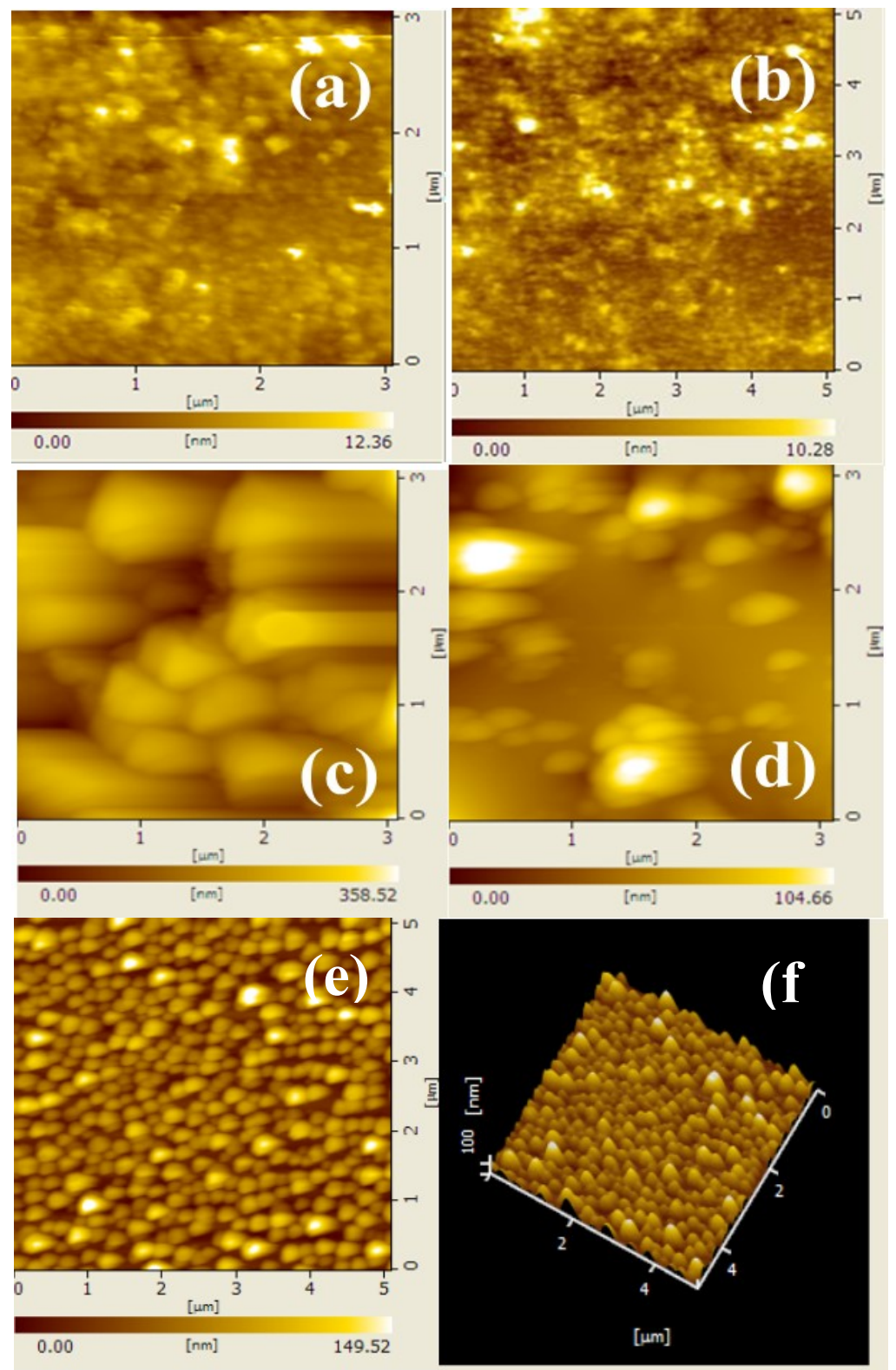


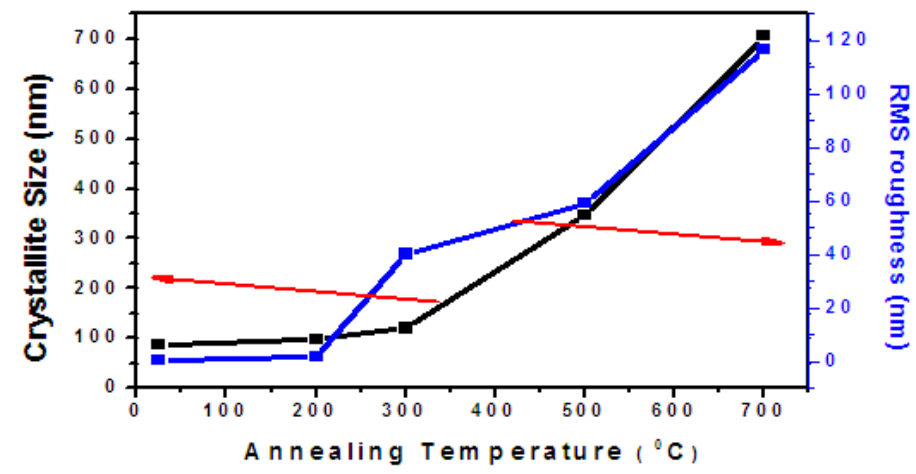

Figure 7

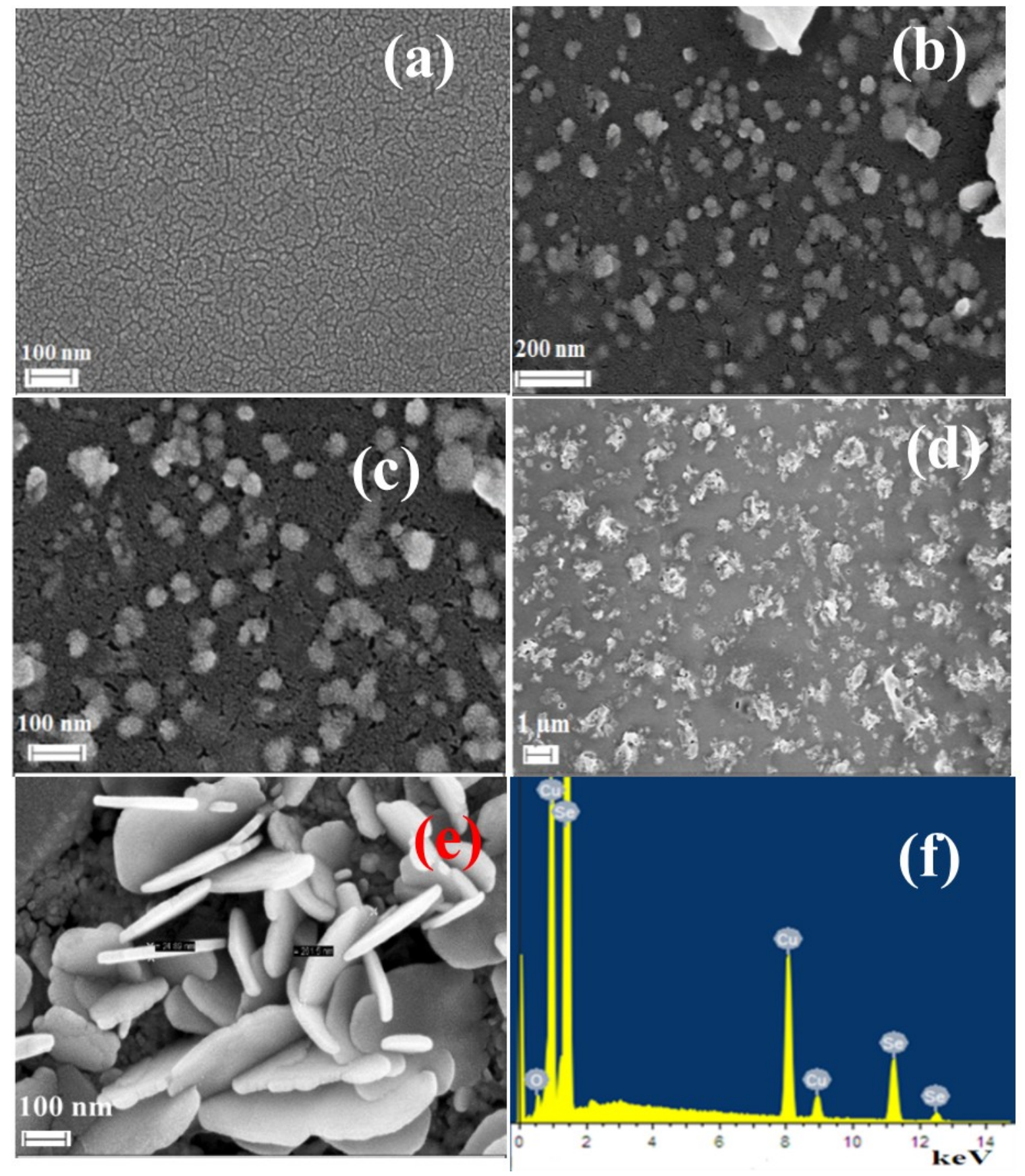




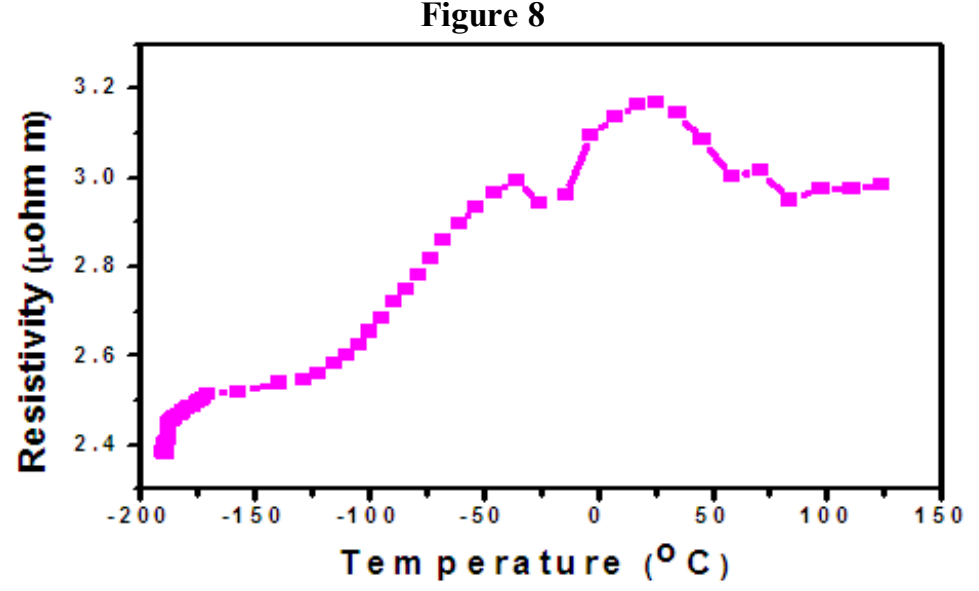

Fig u re 9

List of Tables

\begin{tabular}{|c|c|c|}
\hline SAMPLE $\backslash$ MEASUREMENT & $\begin{array}{c}\text { Crystallite Size } \\
(\mathbf{n m})\end{array}$ & Lattice parameters (nm) \\
\hline Powder & 80.8286 & $\mathrm{a}=1.420, \mathrm{c}=1.725$ \\
\hline Pellet & 24.0130 & $\mathrm{a}=1.414, \mathrm{c}=1.729$ \\
\hline As-deposited Thin Film & 28.0299 & --- \\
\hline Annealed at $200^{0} \mathrm{C}$ & 42.555 & --- \\
\hline Annealed at $300^{\circ} \mathrm{C}$ & 60.7091 & $\mathrm{a}=1.415, \mathrm{c}=1.736$ \\
\hline
\end{tabular}

Table 1

\begin{tabular}{|c|c|c|}
\hline Annealing Temperature ( ${ }^{\mathbf{C}}$ ) & Thickness (nm) & Band Gap (eV) \\
\hline As deposited film & 268 & 1.9482 \\
\hline 200 & 236 & 3.1505 \\
\hline 300 & 157 & 3.5416 \\
\hline 500 & 118 & 3.5961 \\
\hline 700 & 97 & 3.7023 \\
\hline
\end{tabular}

Table 2

\begin{tabular}{|c|c|c|}
\hline $\begin{array}{c}\text { SAMPLE } \backslash \\
\text { MEASUREMENT }\end{array}$ & $\begin{array}{c}\text { Crystallite size } \\
\text { (nanometres) }\end{array}$ & $\begin{array}{c}\text { RMS roughness } \\
\text { (nanometres) }\end{array}$ \\
\hline As-deposited film & 87.63 & 0.6645 \\
\hline Annealed at $200{ }^{\circ} \mathrm{C}$ & 98.8077 & 1.681 \\
\hline Annealed at $300{ }^{\circ} \mathrm{C}$ & 119.9635 & 40.2 \\
\hline Annealed at $500{ }^{\circ} \mathrm{C}$ & 346.755 & 58.92 \\
\hline Annealed at $700{ }^{\circ} \mathrm{C}$ & 704.6096 & 116.63 \\
\hline
\end{tabular}

Table 3 